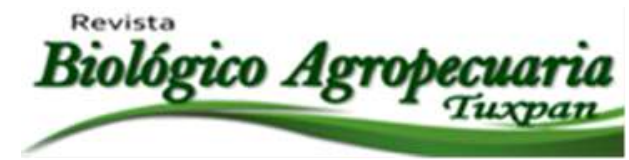

\title{
Distribución weibull para Pinus patula schiede ex schltdl. Et. Cham. En plantaciones forestales comerciales
}

Weibull distribution for Pinus patula schiede ex schltdl. Et. Cham. In commercial forest plantations

Uranga Valencia Luisa Patricia ${ }^{1 凶}$, De los Santos Posadas Héctor Manuel², Quiñonez Barraza Gerónimo², Villarreal Ramirez Víctor Hugo ${ }^{4}$, Quiroz Guzman Tamara ${ }^{5}$

${ }^{1,4}$ Facultad de Ciencias Agrícolas y Forestales. Universidad Autónoma de Chihuahua. Km. 2.5 Carretera Delicias-Rosales, Campus Delicias. C.P. 33000 Apartado Postal 253 Cd. Delicias, Chihuahua, México. ${ }^{2}$ Programa Forestal. Colegio de Postgraduados. Carretera México- Texcoco Km 36.5, Montecillo, Texcoco. C.P. 56230. Estado de México. México. ${ }^{3}$ Campo Experimental Valle del Guadiana, Instituto Nacional de Investigaciones Forestales Agrícolas y Pecuarias (INIFAP). Carretera Durango-Mezquital Km 4.5, 34170 Durango, Durango, México. 5,6Instituto Tecnológico de Delicias, Paseo Tecnológico km 3.5 Carretera a Rosetilla. Cd. Delicias Chihuahua Mexico.

${ }^{凶}$ Autor para correspondencia: luranga@uach.mx

\section{RESUMEN}

Los sistemas de rendimiento que proveen estimaciones de volumen maderable por clase diamétrica (predicción implícita) son una herramienta de alto valor para la planificación del manejo forestal, ya que, al revelar la estructura del rodal, permite definir los tratamientos silvícolas que se pueden aplicar y saber a qué edad y diámetro se encuentra la mayor concentración de volumen. Sin embargo, la Cooperativa Integradora Forestal de Zacualpan, Veracruz (CIFZA) ha establecido 3,000 ha con Pinus patula durante los últimos 14 años, y no cuenta con estudios que permitan estimar productividad o predecir el crecimiento maderable para programar la cosecha. Considerando lo anterior, se desarrolló un sistema en base a distribuciones diamétricas. En su construcción se utilizó información dasométrica derivada de dos remediciones en 50 parcelas permanentes de muestreo de $400 \mathrm{~m}^{2}$; en una crono-secuencia de edades que van de manera compacta de $\operatorname{los} 3$ a los 11 años pero que tienen parcelas aisladas de hasta 21 años. El sistema implícito se desarrolló tomando como base la distribución Weibull de tres parámetros y sugiere que a los 15 años la distribución diamétrica más probable concentra el $45.17 \%$ del volumen de árboles mayores de $25 \mathrm{~cm}$. Evidentemente en plantaciones forestales comerciales se requiere de prácticas silvícolas intensivas y bien programadas a fin de maximizar el potencial de crecimiento durante los primeros 20 años.

Palabras clave: Productividad forestal, volumen, percentiles, clases diamétrica.

\begin{abstract}
The yield systems that provide predicting of timber volume by diametric class (implicit prediction) are a high value tool for forest management planning, since, by revealing the structure of the stand, it allows to define the silvicultural treatments that can be applied and know at what age and diameter is the highest concentration of volume. However, the Cooperativa Integradora Forestal de Zacualpan, Veracruz (CIFZA) has established 3,000 ha with Pinus patula during the last 14 years, and does not have studies to estimate productivity or predict timber
\end{abstract}


growth to schedule the harvest. Considering the above, a system was developed based on diametric distributions. In it's construction, dasometric information derived from two re-uses was used in 50 permanent sampling plots of $400 \mathrm{~m}^{2}$; in a chrono-sequence of ages that go from compact form of the 3 to the 11 years but that have isolated plots of up to 21 years. The implicit system was developed based on the Weibull distribution of three parameters and suggests that at 15 years the most probable diameter distribution concentrates $45.17 \%$ of the volume of trees larger than $25 \mathrm{~cm}$. Evidently in commercial forest plantations intensive and well-planned silvicultural practices are required in order to maximize the growth potential during the first 20 years.

Keywords: Forest productivity, volume, percentiles, diametric clases.

\section{INTRODUCCIÓN}

Lograr un manejo forestal óptimo en plantaciones forestales comerciales (PFC), requiere de estimaciones confiables del rendimiento e incremento en volumen de madera para establecer adecuadamente un plan de operación en el mediano y largo plazo, para ello, es necesario que los silvicultores cuenten con las herramientas cuantitativas adecuadas que les permitan simular y predecir los efectos del manejo a realizar. Lo anterior se lleva a cabo mediante la modelación del rendimiento e incremento con ecuaciones matemáticas que representan los componentes del rodal (Davis et al., 2001, Velázquez et al., 2009; SantiagoGarcía et al; 2013).

El volumen puede estimarse en función de la edad, altura dominante, área basal y el número de árboles; que agregadas como funciones matemáticas interrelacionadas conforman un sistema de crecimiento y rendimiento maderable (SCRM).

\section{MATERIALES Y MÉTODOS}

El estudio se desarrolló en plantaciones de Pinus patula establecidas por la CIFZA, en el municipio de Zacualpan, ubicado en la Huasteca baja del estado Veracruz, México entre los paralelos $20^{\circ} 24^{\prime}$ y $20^{\circ} 37^{\prime}$ de latitud Norte; los meridianos $98^{\circ} 13^{\prime}$ y $98^{\circ} 27^{\prime}$ de longitud
Existen dos tipos de SCRM a nivel de rodal que son ampliamente utilizados, los explícitos e implícitos. Los modelos de distribuciones diamétricas o de predicción implícita, permiten calcular el rendimiento total para obtener un mayor detalle de la distribución diamétrica de la masa forestal $y$ en consecuencia de la distribución de productos maderables en función del tamaño y frecuencia de los árboles (Maldonado y Návar, 2002; Galán et al., 2008; Magaña et al., 2008).

En este estudio se evalúa el enfoque implícito para las plantaciones de la Cooperativa Integradora Forestal de Zacualpan, Veracruz (CIFZA) permitiendo modelar de mejor manera las características dasométricas de las plantaciones. A la fecha la CIFZA cuenta con 3,000 ha plantadas de Pinus patula, pero carece de información silvícola para realizar planificación a mediano y largo plazo.

Oeste.La información se colecto en una de red de 50 sitios de bajo un muestreo sistemático estratificado por edad, en parcelas de forma rectangular de $400 \mathrm{~m}^{2}$, cada uno de los sitios conformado de tres a cinco parcelas por edad, oscilando éstas, entre los 3 y 21 años. 
La predicción implícita del rendimiento actual y su proyección se realizó implementando la metodología sugerida por Clutter et al., (1983); este método considera un enfoque de las distribuciones diamétricas basadas en la función de densidad de probabilidad (fdp) Weibull de tres parámetros, cuya función de densidad de probabilidad es: $f(x)=\left\{\frac{c}{a}\left(\frac{x-a}{b}\right)^{c-a} \times\right.$ $e^{\left[-\left(\frac{x-a}{b}\right)^{c}\right]},(a \leq x \leq \alpha) \quad 0$, de otra forma donde $a$ es el parámetro de localización, $b$ de escala y $c$ de forma. Los parámetros $b$ y $c$ son positivos; mientras que el parámetro $a$, puede tomar valores negativos, positivos o cero, pero se limita a ser positivo para representar una distribución de diámetros y se está relacionando al valor mínimo de la variable. Una expresión analítica cerrada de la función de distribución acumulada está dada por: $f(x)=1-e^{\left[-\left(\frac{x-a}{b}\right)^{c}\right]}$. Esta relación puede establecerse de la siguiente forma: si una población tiene una distribución Weibull, la proporción de la misma con valores superiores a $I$ (diámetro menor de una clase) y menores que $S$ (diámetro superior a una clase) está dada por la expresión: $P(I<x<S)=$ $e^{\left[-\left(\frac{I-a}{b}\right)^{c}\right]}-e^{\left[-\left(\frac{S-a}{b}\right)^{c}\right]}$. Esto se refiere a la probabilidad de que $x$ este ente $I$ y $S$ cuando la función de distribución acumulativa es la Weibull. Cuando los parámetros de la distribución Weibull ya han sido estimados, se puede obtener la proporción de árboles por clase de diámetro, mediante la aplicación de la expresión anterior. Para el estudio se consideraron categorías de $5 \mathrm{~cm}$. Como parte esencial de la predicción del rendimiento maderable con distribuciones diamétricas, es necesario hacer una estimación del número de árboles sobrevivientes por hectárea $(\mathrm{N})$ a través del tiempo. Para generar estimaciones de distribuciones diamétricas, se estimaron los tres percentiles centrados en el diámetro cuadrático. Los percentiles 65, 95 y 0 resultaron eficientes. Así, las ecuaciones de predicción de percentiles tomaron la siguiente forma: $p 65=\alpha_{1} \times$ $D q^{\beta_{1}} ; p 95=\alpha_{2} \times p 65^{\beta_{2}} ; p 0=\alpha_{3} \times p 65^{\beta_{3}}$; donde $p 65$, $p 95$ y $p 0$ son los percentiles $65,95 \mathrm{y}$ 0 de la distribución diamétrica; $\alpha_{i}$ y $\beta_{i}$ son los parámetros a estimar y $D q$ es el diámetro cuadrático. Los parámetros de las ecuaciones que predicen los percentiles 65, 95 y 0 se ajustaron utilizando el método de mínimos cuadrados ordinarios. El diámetro cuadrático $(\mathrm{Dq}, \mathrm{cm})$ corresponde al diámetro del árbol de área basal media, por lo que: $D q=\sqrt{\frac{40000}{\pi} \times \frac{A B}{N}}$. El volumen del fuste total para cada árbol se calculó mediante la siguiente ecuación tipo Schumacher y Hall (1933): $V=0.000114 \times$ $D^{1.718158} \times H^{0.939592}$ generada para la zona donde $D$ es el diámetro normal (a $1.30 \mathrm{~m}$ ) y $H$ es la altura total del árbol en m. La altura fue obtenida con una curva altura-diámetro ajustada para tal fin $A=1.32+0.5823 \times D$. Los percentiles se utilizan para la recuperación de parámetros de una distribución Weibull bajo el criterio de Piennar et al., (1996), dichos parámetros fueron estimados por el método de momentos; obteniendo el parámetro de localización $a$, con la siguiente expresión:

$$
=\left\{\begin{array}{c}
\text { si } p 0 \geq 2.5 \text { entonces } \hat{a}=p 0-2.5 \\
\text { De otra forma } \\
\hat{a}=\frac{p 0}{2.5}
\end{array}\right.
$$

Una vez conocido el parámetro $a$ es posible determinar el parámetro de forma; es decir $c$, de la siguiente manera: $\hat{c}=$ $\frac{\ln [-\ln (1-0.95) /-\ln (1-0.65)]}{\ln [(p 95-\hat{a}) /(p 65-\hat{a})]} \quad \mathrm{Y}$ por último, se calcula el parámetro de escala $b$, ya que requiere los valores de $a$ y $c$; estimándose 
como: $\hat{b}=-\hat{a} \times\left(\Gamma_{1} / \Gamma_{2}\right)+\left[\left(\hat{a} / \Gamma_{2}\right)^{2} \times\left(\Gamma^{2}{ }_{1}-\right.\right.$ $\left.\left.\Gamma_{2}\right)+\left(D q^{2} / \Gamma_{2}\right)\right]^{0.5}$

donde: $\ln ($.$) es el logaritmo natural; \Gamma_{1}=$ $\Gamma\left(1+\frac{1}{\hat{c}}\right) ; \Gamma_{2}=\Gamma\left(1+\frac{2}{\hat{c}}\right) ; \quad \Gamma($.)es la función Gamma; las demás variables ya fueron definidas.Si bien este no es el único método de generar un SCRM implícito ha demostrado ser más que adecuado para plantaciones coetáneas.

Para conocer la bondad de ajuste de la fdp Weibull a las estructuras diamétricas de rodales de $P$. patula, se utilizó la prueba de Kolmogorov-Smirnov $(K S)$. Esta prueba permite detectar la máxima diferencia entre la distribución (real) y la estimada (teórica), lo que implica que dicha prueba solo es válida si los parámetros de la fdp Weibull son conocidos para cada distribución diamétrica (Torres-Rojo et al., 1992). En la prueba de $K S$ se utilizaron distintos niveles de significancia $(\alpha=0.05,0.10$ y 0.20$)$ para contrastar el número de pruebas de parcelas no ajustadas a la fdp Weibull de acuerdo a cada criterio

conocer la bondad de ajuste de la fdp Weibull a las estructuras diamétricas de los rodales de Pinus patula estimados.

En el cuadro 1, se presentan los resultados obtenidos de la prueba KS bajo diferentes niveles de significancia. Dicha prueba permito

Cuadro 1. Parcelas rechazadas por su falta de ajuste a la fdp Weibull considerando diferentes niveles de significancia

$\alpha$

Proporción ${ }^{\mp}(\%)$

Numero de distribuciones rechazadas

\begin{tabular}{lcc}
\hline 0.05 & 6 & 6 \\
0.10 & 9 & 9 \\
0.20 & 12 & 12 \\
\hline
\end{tabular}

${ }^{ }$Proporción calculada a partir de 100 distribuciones diamétricas.

En la prueba normalmente se emplea un nivel de significancia de $0.05(\alpha=0.05)$, aunque autores como Álvarez y Ruiz (1998), Pece et al., (2000), García et al., (2002) y Gorgoso et al., (2007) utilizaron un nivel del $\alpha=0.20$, dando con ello un mayor nivel de exigencia al contraste. Al respecto Pece et al., (2000) mencionan que este nivel reduce las desviaciones mínimas permitidas para el no rechazo de la concordancia. Considerando el criterio más exigente $(\alpha=0.20)$, la fdp Weibull se ajustó exitosamente en casi el $90 \%$ de los casos a la distribución diamétrica observada.

Los parámetros estimados, así como los criterios estadísticos de evaluación del ajuste de las ecuaciones que predicen los percentiles de distribución diamétrica de las plantaciones de Pinus patula se muestran en el cuadro 2. 
Cuadro 2. Criterios de ajuste y parámetros estimados de las ecuaciones que predicen los percentiles de la fdp de Weibull en las plantaciones de pino patula en Zacualpan, Ver.

\begin{tabular}{|c|c|c|c|c|c|c|c|}
\hline \multirow[t]{2}{*}{ Modelo } & \multirow[t]{2}{*}{ Parámetro } & \multirow[t]{2}{*}{ Estimación } & \multirow{2}{*}{$\begin{array}{c}\text { Error } \\
\text { Estándar }\end{array}$} & \multirow{2}{*}{$\begin{array}{c}\text { Valor } \\
\mathrm{t}\end{array}$} & \multirow{2}{*}{$\begin{array}{l}\text { Aprox. } \\
\operatorname{Pr}>|t|\end{array}$} & \multicolumn{2}{|c|}{$\begin{array}{c}\text { Estadísticos de } \\
\text { ajuste }\end{array}$} \\
\hline & & & & & & RMSE & $\mathrm{R}^{2} \mathrm{adj}$ \\
\hline & $\square \square$ & 0.995684 & 0.0165 & 60.23 & $<.0001$ & & \\
\hline p65 & $\square \square$ & 1.028714 & 0.00563 & 182.63 & $<.0001$ & 0.3732 & 0.9975 \\
\hline \multirow[t]{2}{*}{ p95 } & $\square \square$ & 1.542097 & 0.0791 & 19.5 & $<.0001$ & 1.5269 & 0.9732 \\
\hline & $\square \square$ & 0.942295 & 0.0170 & 55.3 & $<.0001$ & & \\
\hline \multirow[t]{2}{*}{$\mathrm{p} 0$} & $\square \square$ & 0.07054 & 0.0165 & 4.27 & $<.0001$ & 1.8640 & 0.8065 \\
\hline & $\square_{\square}$ & 1.553461 & 0.073 & 21.28 & $<.0001$ & & \\
\hline
\end{tabular}

RMSE: raíz del error medio cuadrático; $\mathrm{R}^{2}$ adj: coeficiente de determinación ajustado

Los percentiles 65 y 95 se estimaron eficientemente (Cuadro 2), debido a que dichos parámetros son dependientes del diámetro cuadrático del rodal, por lo que al proyectar dicha variable en el tiempo se conoce el valor de estos parámetros a una edad de interés; dichos percentiles fueron útiles para la determinación del parámetro $c$. Existen varios trabajos donde se ajustan diferentes pares de percentiles, sin embargo; concuerdan en el empleo de un percentil superior o igual a $90 \%$. Una vez conocidas las variables anteriores para el sitio promedio (IS 19) a una edad base o de referencia de 15 años, la densidad inicial es de 1,100 árboles por hectárea y Dq calculado de manera indirecta, se estimaron los parámetros de la fdp Weibull y se obtuvo la proporción de árboles por categoría (Figura 1).

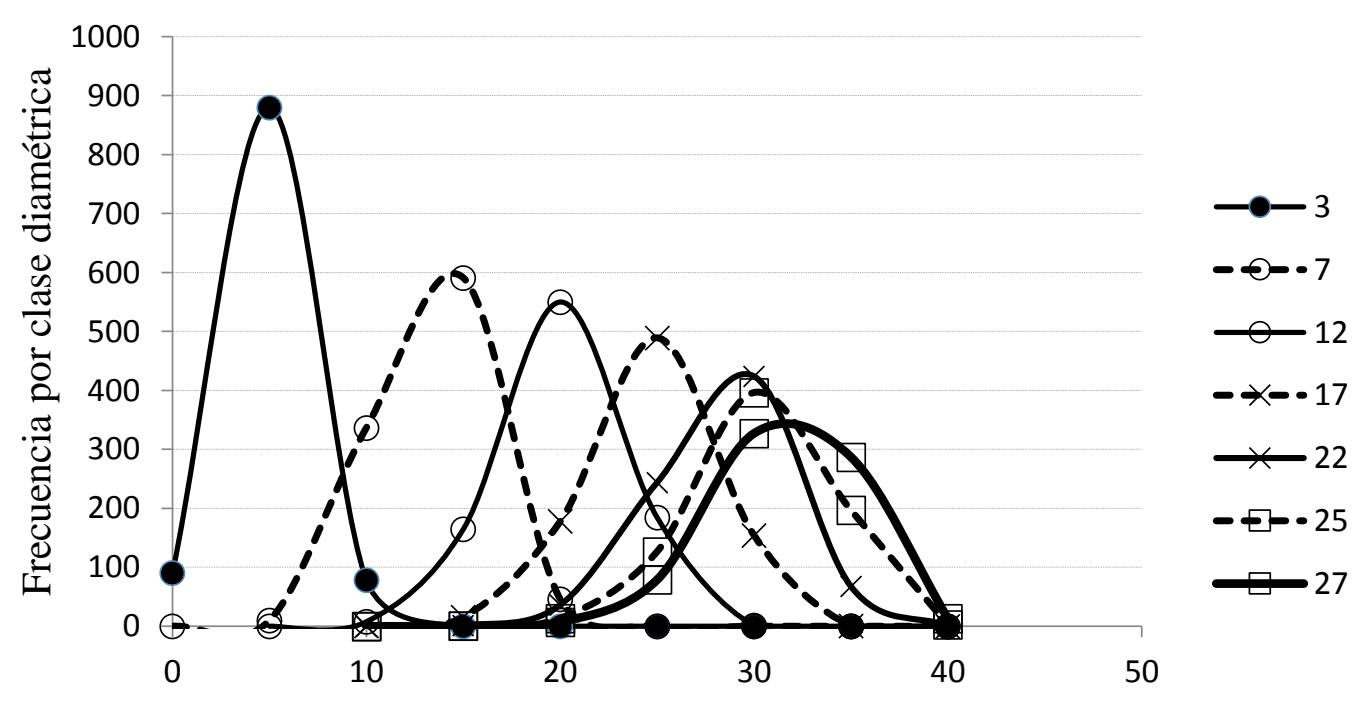

Clases Diamétricas 
Figura 1. Proporción de árboles por hectárea por categoría diamétrica basada en dfp Weibull para las edades $3,7,12,17,22,25$ y 25 años, para el índice de sitio promedio (19 m), edad de referencia de 15 años y una edad inicial de 1,100 árboles.

En la figura 2 se muestra el rendimiento volumétrico por categoría diamétricas y volumen total del sistema implícito

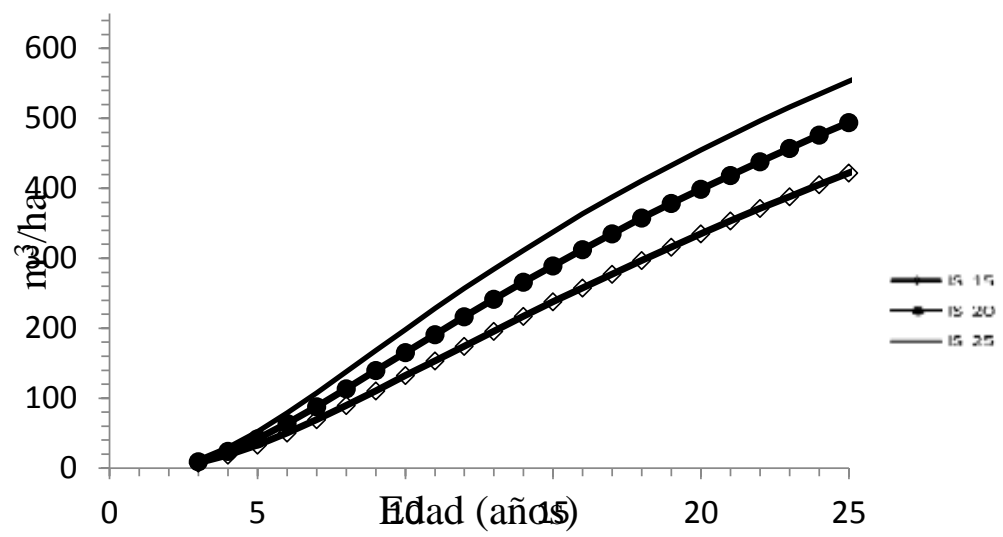

Figura 2. Curvas de rendimiento volumétrico y para el modelo implícito (líneas continuas) para los IS $(15,20$, y 25) para la especie de Pinus patula en Zacualpan, Veracruz, a una edad base de 15 años.

El volumen estimado dependiendo del IS tiende a ser mucho más optimista en IS más ricos mientras que en índices de sitios promedio y bajo los rendimientos existe una diferencia de $50 \mathrm{~m}^{3} / \mathrm{ha}$.

Los sistemas implícitos están diseñados para estimar de manera dinámica el cambio en la distribución de productos con la edad al combinar tanto los diámetros mínimos de corta por producto con las especificaciones de las puntas no comerciales. En este caso para ilustrar la flexibilidad del sistema se propone dividir el volumen del IS medio, densidad inicial de 1,100 árboles y tres categorías de clases diamétricas: Árboles de categoría $15 \mathrm{~cm}$ y menores, árboles de categorías 20 a $30 \mathrm{~cm}$ y árboles de categoría $30 \mathrm{~cm}$ y mayores (Figura 3). 


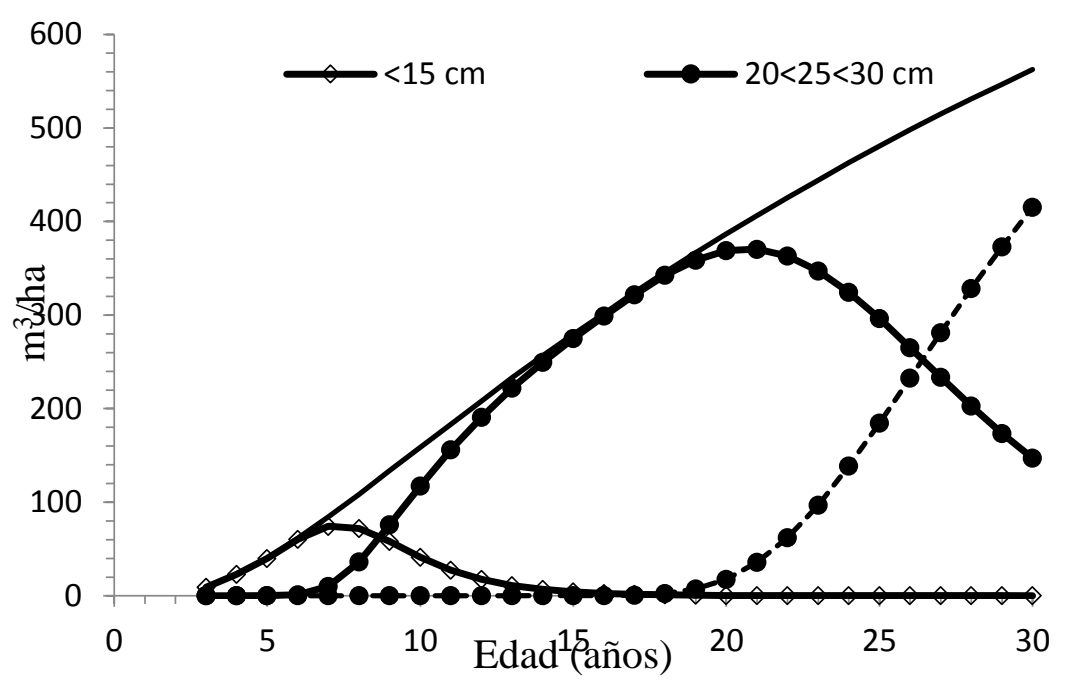

Figura 3. Rendimiento en volumen maderable para las categorías establecidas basados en la fdp Weibull para un índice de sitio promedio (19 m) a una edad base de 15 años con una densidad inicial de 1,100 árboles.

Es interesante notar que los cambios en el volumen se dan de manera acelerada antes de los 15 años. Después de los 15 y hasta casi los 20 años la totalidad del volumen se distribuye entre arboles de categorías 20 a $30 \mathrm{~cm}(17.5$ a 32.5 $\mathrm{cm})$. Es después de los 20 años que la mayoría del volumen empieza a concentrase en los arboles de categoría $30 \mathrm{~cm}$ y mayores.

Una forma de reclutar una mayor cantidad de volumen en categorías diamétricas mayores (pensando sobre todo en la producción de material de aserrío) es disminuyendo la densidad inicial. En este caso se propone una densidad inicial de 800 árboles. Al simular el rendimiento total en las categorías definidas (Figura 4) se aprecia que el volumen proveniente de árboles mayores de categoría 30 empieza a aparecer a los 15 años. El rendimiento total en volumen para árboles mayores de categoría $30 \mathrm{~cm}$ se ve beneficiado de esta menor densidad sin impactar notablemente el rendimiento total.

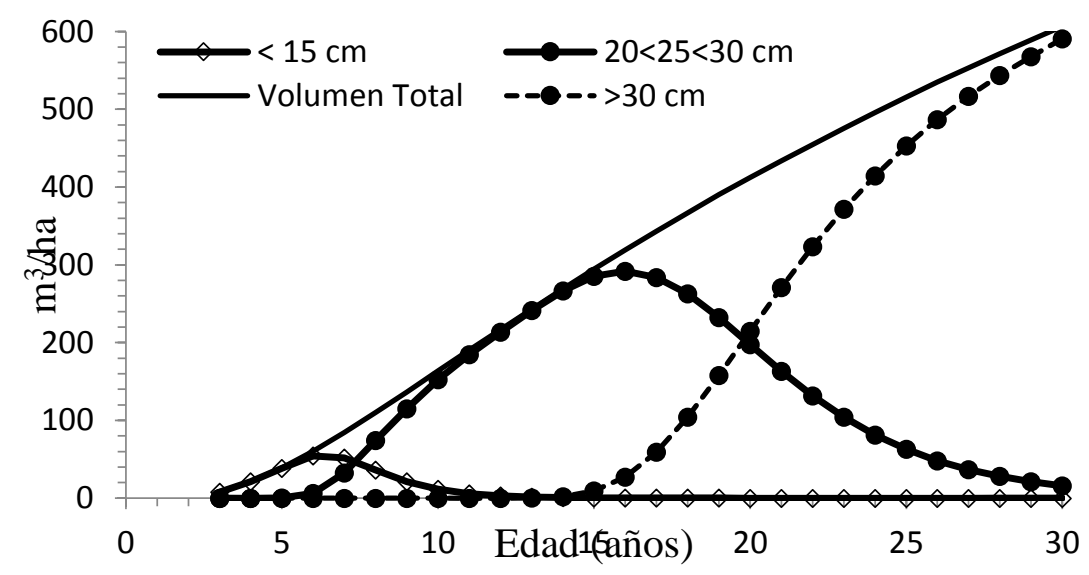


Figura 4. Rendimiento en volumen maderable para las categorías establecidas basados en la fdp Weibull en un índice de sitio promedio (19 m) a una edad base de 15 años con una densidad inicial de 800 árboles.

\section{CONCLUSIONES}

El sistema implícito permitió obtener buenas predicciones del rendimiento maderable por categoría diamétrica a nivel de superficie. El poder representar la estructura diamétrica a medida que cambia su edad debe permitir una mejor planificación en el manejo de los rodales de Pinus patula en la región de Zacualpan; en conjunto y a medida que se mejores las prácticas silvícolas se podrán incorporar mejores fuentes de semilla, así como mejores prácticas de preparación del terreno, y labores silvícolas intensivas, sin duda cambiara el rendimiento y estructura diamétrica. Sin embargo, los resultados obtenidos sugieren que el potencial de la zona para la producción maderable vía PFC es promisorio y requeriría para su éxito como actividad económicamente rentable de una correcta planificación.

\section{LITERATURA CITDA}

Álvarez G, J. G., y A. D. Ruíz G. 1998. Análisis y modelación de las estructuras diamétricas de Pinus pinaster Ait. en Galicia. Investigación Agraria: Sistemas y Recursos Forestales 7: 123-137.

Borders, B. E., R.A. Souter, R. L. Bailey, and K. D. Ware. 1987. Percentile-based distributions characterize forest stand tables. Forest Science 33: 570-576.

Clutter J. L. 1963. Compatible growth and yield models for loblolly pine. Forest Sciencie 9: 354-371.
Clutter J. L., J. C. Forston., L. V. Pienaar., G. H. Brister., R. L. Bailey. 1983. Timber Management: A Quantitative Approach. John Wiley \& Sons, Inc. New York. 333 p.

Davis L. S., K. N. Johnson., P. S. BettingeR and T. E. Howard. 2001. Forest Management. $4^{\text {th }}$ ed. McGraw-Hill Series in Forest Resources. 804 p.

Galán L. R., H. M. De los Santos P. y J. I. Valdez H. 2008. Crecimiento y rendimiento maderable de Cedrela odorata I. y Tabebuia donnell-smithii Rose en San José Chacalapa, Pochutla, Oaxaca. Madera y Bosques 14: 65-82. https://doi.org/10.21829/myb.2008.1421

García, G. C., Cañadas, y G. Montero. 2002. Modelización de la distribución diamétrica de las masas de Pinus pinea L. de Valladolid (España) mediante la función Weibull. Investigación Agraria: Sistema y Recursos Forestales 11 (2): 263-282.

Gorgoso, J. J., J. G. Álvarez G., A. Rojo, and J. A. Granadas-Arias. 2007. Modelling diameter distributions of Betula Alba L. stands in northwest Spain with the two parameter Weibull function. Investigación Agraria: Sistemas y 
Recursos Forestales 16 (2): 113-123. https://doi.org/10.5424/srf/2007162-

Magaña T. O. S., J. M. Torres R., C. Rodríguez F. H. Aguirre D., y A. M. Fierros G. 2008. Predicción de la producción y rendimiento de Pinus rudis Endl. en Aloapan. Oaxaca. Madera y Bosques 14: 5-19. https://doi.org/10.21829/myb.2008.

Maldonado D. A. y J. Návar Ch. 2002. Ajuste y predicción de la distribución Weibull a las estructuras diamétricas de plantaciones de pino de Durango, México. Madera y Bosques 8: 61-72. https://doi.org/10.21829/myb.2002.8113

Pece M. G., C. G. De Benítez, y M. J. De Galíndez. 2000. Uso de las funciones Weibull para modelar distribuciones diamétricas en una plantación de Melina azedarach. Revista Forestal Venezolana 44(2): 49-52.

Piennar, L.V., B. D. Shiver and J.W. Rheney. 1996. Yield prediction for mechanically site-prepared slash pine plantations in The Southeastern Coastal Plain. PMRC Technical Report 1996-3. Daniel B. Warnell School of Forest Resources. The University of Georgia.

Santiago-García, W., H. M. De los SantosPosadas, G. Ángeles-Pérez, J. R. ValdezLazalde y G. Ramírez-Valverde. 2013. Sistema compatible de crecimiento y rendimiento para rodales coetáneos de Pinus patula. Revista Fitotecnia Mexicana. 36 (2): 163-172.

https://doi.org/10.35196/rfm.2013.2.163

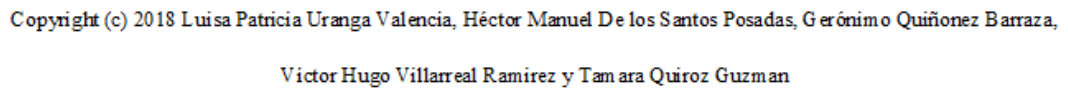

Victor Hugo Villarreal Ramirez y Tam ara Quiroz Guzman

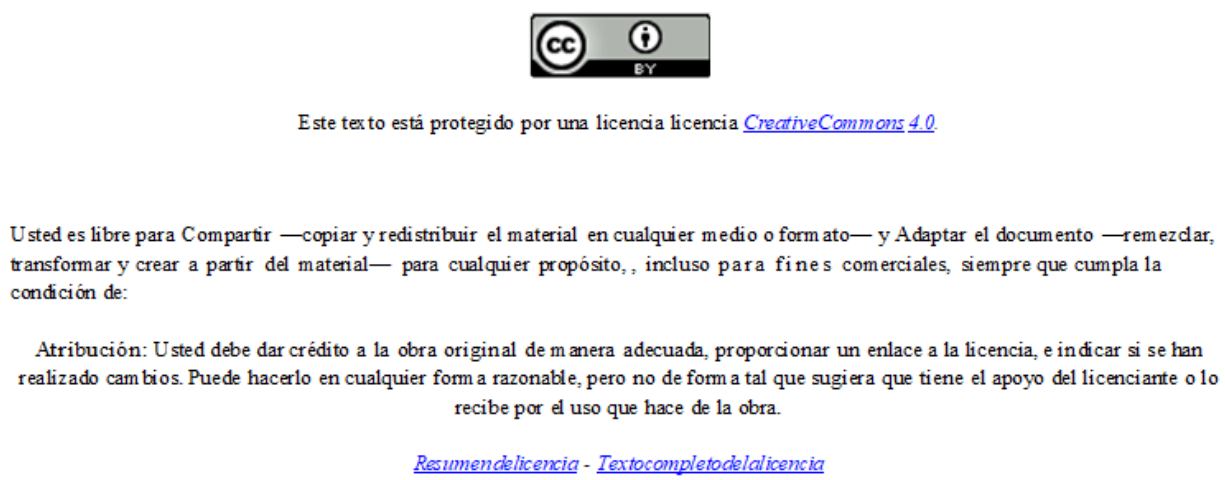

